\title{
La Revista de América como vínculo de la cultura letrada latinoamericana: contexto y usos del pasado en el desarrollo de la idea de América Latina
}

Perla Itzamná Navarro Sánchez: Alexander Betancourt Mendieta*

\section{Resumen}

Este trabajo analiza la influencia que tuvo la cultura letrada en la fundación y formación de la Revista de América que dirigió Germán Arciniegas. La Revista de América surgió como un medio para reflexionar sobre el subcontinente como conjunto y su papel en el mundo después de los conflictos bélicos mundiales. Los contenidos de la Revista trazaron ideas para tomar decisiones sobre lo que América Latina representaba como continente y su relación con el resto del mundo en la coyuntura de la posguerra.

Palabras clave: Cultura letrada - revistas culturales - América Latina

\section{Summary}

This text studies the influence of the written culture in the publication of the Revista de América directed by German Arciniegas. The Revista de América represented a place to think about the subcontinent and its central role after world war conflicts. Its contents made a way to take decisions about what Latin America represents as a continent and its relation with the rest of the world in the conjuncture of the postwar period.

Keywords: Written culture - cultural journals - Latin America

* Universidad Autónoma de San Luis Potosí. E mail: itzamnanavarro@gmail.com

** Universidad Autónoma de San Luis Potosí. E mail: alekosbe@uaslp.mx 


\section{Introducción}

La importancia de analizar y estudiar el desarrollo de la cultura letrada en América Latina radica en el carácter fundamental que tuvieron los hombres de letras en la construcción, formación y consolidación del pasado nacional, pero también en la manifestación de la preocupación sobre el "ser" y "devenir" de todo un conjunto de países que desde fines del siglo XIX e inicios del XX fueron considerados en algunos esfuerzos intelectuales y políticos como parte de un sólo conjunto cultural. Por eso, abordar cómo el uso de la palabra escrita facilitó el desarrollo de una idea una América Latina como un conjunto homogéneo vinculado por elementos comunes como la lengua y la historia, de esta misma forma, permitirá explicar cómo estos elementos comunes justificaron actividades como la escritura, el impulso de agendas de tipo político, el impulso de publicaciones periódicas que generaron un campo de acción cultural bastante amplio.

Para estudiar la cultura letrada en América Latina es necesario considerar la ausencia de espacios específicos para la formación profesional dentro del ámbito de la educación superior y los efectos que esto tuvo en las prácticas de la escritura y la difusión de sus contenidos. Esta situación propició el uso de espacios de sociabilidad como los cafés, las conferencias, círculos científicos y literarios que sirvieron como los lugares de referencia para desarrollar la parte material que dio vida y soporte a las prácticas del mundo letrado. También es necesario tener en cuenta a la materialidad del trabajo letrado que se refiere a los textos propiamente dichos, y a sus soportes, ya fueran panfletos, periódicos, diarios o revistas. (Granados, 2017:72).

Hilda Sábato (2008) afirma sobre la sociabilidad letrada y sus instituciones en el siglo XIX, que estos dos elementos permiten dilucidar cuáles de estas pautas se instituyeron de tal forma que continuaron hasta el siglo XX, y cuáles de ellas sufrieron cambios significativos. La autora refiere que en las prácticas asociacionistas de ciertos grupos de hombres letrados en la segunda mitad del siglo XIX se encontraba la tendencia de la protección de los intereses grupales, la defensa de las opiniones generadas desde su interior, pero también cumplían ya con una característica importante que era la de entretejer toda una red de mecanismos que atravesaban directamente a la sociedad, fuese letrada, política o incluso analfabeta. 
Los espacios que estos hombres letrados procuraron crear sirvieron para mantener las conexiones con la vida pública latinoamericana y también eran los escaparates para que se hicieran publicaciones de prensa periódica. (Sábato, 2008: 387).

La mayor parte de las publicaciones eran de muy corta vida y escasa circulación, limitada con frecuencia a los sectores más acomodados de la población. De todas maneras, su influencia trascendia el circulo estricto de quienes las compraban y podian leerlas.

En primer lugar, porque funcionó como un espacio de expresión política, tanto cuando el conflicto se expresaba directamente en sus páginas, como cuando la censura admitía sólo el discurso oficialista. También, porque los periódicos no eran leídos de manera exclusivamente individual. Así, en cafés y en sociedades de lectura se generaban sesiones de discusión de los articulos de la prensa, mientras que algo semejante ocurría en las pulperías y en las chicherías, o aun en la calle, donde no faltaba quien leyera en voz alta para beneficio de la mayoría analfabeta. (Sábato, 2008: 393).

Las publicaciones, en particular las revistas culturales, tenían rasgos materiales que permitían diferenciarlas de otro tipo de publicaciones a partir de aspectos como la paginación, el diseño, la calidad de los materiales. Quizás uno de los aspectos que ayudaban a plantear diferencias entre las publicaciones era la disposición de las portadas, si es que hay alguna imagen o sólo presentaban letras, puesto que desde el uso de ambas cosas puede considerarse algunos anticipos sobre los contenidos de la publicación. También es necesario considerar detalles como la impresión y encuadernación que permiten ver qué condiciones fueron necesarias para la producción, puesto que este aspecto puede dar cuenta de la pobreza o el apoyo monetario a un proyecto editorial. El conjunto de estos factores, desde la integración del grupo de hombres de letras hasta los rasgos materiales de la publicación facilitaron el surgimiento y la continuidad de un campo de actividades que tuvieron como resultado la formulación de un canon de escritura que posicionó a los hombres de letras vinculados a estos procesos en individuos como ejes trasformadores de la sociedad para modernizarla y civilizarla. (Pita, 2015: 181).

\section{Latinoamericanismo y cultura letrada}

El conjunto de las prácticas de los hombres de letras en torno a la producción, difusión y lectura de sus textos se le denomina cultura letrada. La cultura letrada 
implica, entonces, la creación de un ambiente que permite el uso de conceptos, hábitos, temáticas en común para el grupo de personas que participan de ese mismo entorno.

(...) la escritura como actividad intelectual, al carecer de espacios institucionales especificos para su desenvolvimiento especializado, entra a formar parte de la cultura letrada que no se caracteriza por la precisión de sus límites y campos de acción, y confluyen en las tareas asumidas por los hombres de letras. (Betancourt, 2018: 13).

El carácter difuso de sus funciones, tocaban al mismo tiempo, la vida política y la alta esfera social, lo que les daba legitimidad a los hombres de letras como especialistas en temas de cultura y opinión sobre los principales sucesos nacionales como internacionales. Si bien desde el siglo XIX las actividades políticas de las que se ocuparon los hombres de letreas sirvieron como base de importantes decisiones en términos de gobierno, también es primordial destacar el papel central que tuvieron en la formación de redes trasnacionales que permitieron la configuración de una idea propia sobre el pasado, presente y devenir del subcontinente y de cada uno de los Estados y las naciones que lo conformaban. A grandes rasgos, esta forma de relación entre la producción letrada y su entorno político continuó hasta bien entrado el siglo XX.

En América Latina, la producción de textos durante el siglo XX también se vio influenciada por los acontecimientos de las últimas décadas del siglo XIX como la victoria de Estados Unidos sobre España en 1898, que dejó al descubierto el poder avasallador que comenzaba a concentrar el país del norte. A partir de estos acontecimientos, la visión que se tuvo desde América Latina con respecto a la relación que se entretejía entre los dos hemisferios del continente no se hizo esperar en los textos producidos desde el subcontinente. La visión que se construyó buscaba resaltar las diferencias que se tenían con respecto a Estados Unidos desde un enfoque de "raza" y "cultura", en donde Estados Unidos representaba la vida material y de consumo sin freno, mientras que América Latina se visualizaba como la heredera de la cultura y valores espirituales europeos como se evidencia en trabajos como el famoso Ariel (1900) de José Enrique Rodó, quien sentó las bases de la comprensión de América en las primeras décadas del siglo XX, ideas que a su vez 
fueron acogidas por otros hombres de letras como Pedro Henríquez Ureña con obras como "La utopía de América” (1925), Francisco García Calderón con "La creación de un continente (1912), Rufino Blanco Fombona con "La evolución política y social de Hispano-América” (1911), entre otros. (Deves, 1997: 18-25).

Las consideraciones sobre la unidad continental de América Latina tuvo un clima favorable en la coyuntura que representaron los festejos del Centenario de las Independencias porque en dichas conmemoraciones se reavivó el interés para hacer énfasis en los rasgos comunes del subcontinente como, por ejemplo, las raíces hispánicas en términos raciales, así como el vínculo con el Viejo Continente, considerado como el seno de "las buenas costumbres" y "la civilización" en contraposición con la lógica de mercado de Estados Unidos. También la importancia de esta coyuntura se encuentra en la relación que tuvo el impulso de una serie de proyectos de índole cultural generados para la conmemoración de las Independencias. Estos van desde la formación de colecciones documentales del periodo que pudieran visibilizar el pasado nacional, la elaboración de monumentos, construcción de edificios, avenidas, museos que mostraran los elementos propios de las historias patrias y también la publicación de textos que enlazaran el pasado nacional con las políticas actuales y la participación latinoamericana en el contexto global. (Malosetti, 2010: 466).

\section{El siglo XX: rasgos de continuidad en la cultura letrada y las revistas culturales}

El cambio de siglo no representó en sí mismo cambios radicales en las prácticas de la sociabilidad letrada y sus productos. Si bien algunas temáticas, como por ejemplo aquellas centradas en la narración del pasado nacional, desaparecieron o disminuyeron en presencia e interés, y surgieron otros temas nuevos que se formularon a través de disertaciones con referencias a situaciones del contexto internacional. Se mantuvieron ciertas formas de presentar sus opiniones políticas y culturales, así como también textos a manera de disertación teórica con una presentación y escritura de tipo literaria. Sobre este último punto, los escritos que 
producían buscaban no sólo informar o generar opinión, sino que también tenían como consigna ser bellos textos para leer, por lo que debían mostrarse con cierto valor literario y estético. $\mathrm{Al}$ ser textos que tenían la forma y estructura de una lectura amena y bella, garantizaba la difusión y, por ende, el carácter educativo para una ciudadanía en proceso de construcción. Este punto era crucial, pues a los hombres de letras se les consideraba productores de sentido, guías de sus comunidades y potenciales agentes de cambio social y político. Dicha visión sobre sus deberes intelectuales continuó incluso después de la década de los 1960, a pesar de la creciente profesionalización en el campo de las ciencias sociales. (Betancourt, 2013: 149).

Los cambios en los procesos de recepción de los materiales escritos producidos por los hombres de letras estaban vinculados con las transformaciones y las políticas educativas que buscaron cubrir la mayor parte de la población. Hasta el siglo XIX y en parte del siglo XX, el uso de la palabra escrita y de la lectura estuvo constreñido sólo a algunos sectores de la población. Sin embargo, con la implementación y creación de centros de instrucción pública desde finales del siglo XIX y ya en las primeras décadas del XX el mercado lector se diversificó, no con ello el interés en la lectura de literatura ni de ensayos. De hecho, hay que destacar que, a pesar de los esfuerzos, la población seguía siendo principalmente analfabeta, aunque con nuevos intentos de masificar la lectura. Por citar algunos ejemplos de estas empresas se encuentran los esfuerzos de los gobiernos de Colombia llevados a cabo entre 1930 y 1946 como la fundación de Misiones Culturales y Bibliotecas Aldeanas, que en conjunto eran proyectos que buscaron amplificar la alfabetización y por ende las prácticas lectoras. (Guzmán, 2016: 186). Este nuevo brío en la lectura impulsó mucho más las prácticas editoriales que propiciaron la fundación de periódicos, y revistas en mayor escala. A pesar de esto, la escritura no alcanzó el grado de profesionalización hasta la segunda mitad del siglo XX, por lo que estos medios impresos fueron los principales medios de expresión y de consolidación de un canon escriturario a seguir, que a su vez marcaron las pautas y temáticas que finalmente llegaban al público lector. (Altamirano, 2008: 10).

Ahora bien, en torno a los textos generados desde el campo de las letras, la forma más común de manifestar las ideas fue a través del ensayo, que representó la forma 
más recurrente de manifestar la visión que sobre América Latina construían los escritores. Igualmente, a través de la escritura de los ensayos fue que se generó el campo adecuado para llevar a cabo debates, intercambios y el uso de un lenguaje común entre quienes formaban los círculos de hombres letrados. Las publicaciones como las revistas dieron un espacio material a estas disertaciones, su periodicidad posibilitó también el intercambio rápido y continuo de opiniones. (Bottinelli, 2019: 31).

Una revista es una publicación que se emite de manera regular con cierta periodicidad ya sea semanal, quincenal, mensual, bimensual o semestral. Esto variará según los objetivos mismos de la revista, así como de las condiciones materiales que faciliten su impresión, diseño y divulgación. Ahora bien, definir "revista cultural" permitirá reconocer su importancia en el ámbito de la cultura letrada latinoamericana. Según menciona Beigel:

Nos referimos a las revistas, -(como)- puntos de encuentro de trayectorias individuales y proyectos colectivos, entre preocupaciones de orden estético y relativas a la identidad nacional, en fin, articulaciones diversas entre politica y cultura que han sido un signo distintivo de la modernización latinoamericana. (...) Tienen la particularidad de que se trata de emprendimientos que estuvieron atados (...) a coyunturas históricas complejas, pero bien recortadas. Pertenecen a una especie de bisagra histórica: una etapa signada por distintas formas de revolución que auguran un cambio de época. (Beigel: 2003: 106).

Las revistas culturales formaron un punto de confluencia y de intercambio de ideas importante durante la primera mitad del siglo XX y las primeras décadas después del periodo posguerra a falta de espacios físicos o institucionales claramente definidos. De hecho, puede considerarse que las revistas fungieron como importantes medios de difusión e interacción que permitieron la continuidad de las prácticas comunes a la cultura letrada, y que, para el caso de América Latina, facilitó la formación y continuidad de ese campo cultural letrado durante varias décadas. También sirvieron como espacios propicios al debate a falta de sitios específicamente definidos para los debates académicos e inclusive tomaron carácter militante ante los acontecimientos políticos y sociales tanto nacionales como globales. (Beigel, 2003: 108).

En cuanto a las revistas, debe acentuarse su valor como fuentes históricas en función de que también permiten reconstruir la forma en la que estas sociedades letradas 
interactuaban entre sí, al mismo tiempo que facilitan ubicar cómo se observaban en su momento los diversos acontecimientos que se desarrollaban, porque estas por lo regular emergen en las coyunturas. Igualmente, dentro de sus funciones principales, se encontraba la de cambiar su entorno cultural. Las revistas llevan en sus contenidos y en sus prácticas de producción y circulación la impronta de la coyuntura en la cual se generaron y justamente sus textos buscan analizar y modificar el statu quo en el seno de esas rupturas. Es así, como las opiniones de los hombres letrados sobre el panorama que viven se plantean en las revistas, es en ellas en las que se procura esclarecer dudas, generar debates y poner a juicio las distintas posturas sobre políticas y también sobre la noción que estos individuos tuvieron sobre el concepto de cultura misma. La revista debe ser considerada entonces como un instrumento que generaron los grupos letrados para conseguir acercarse al entorno político de su época y hacer desde estos campos política cultural. (Sarlo, 1992: 9).

Las revistas son en sí mismas una fuente para indagar cómo se organizaban discursos de distinta índole en torno a un sistema de relaciones entre hombres de letras que se vinculaban a través de afinidad generacional e ideológica, y que por ello generaron una amplia red de comunicación no sólo cultual sino política, tanto a nivel nacional, subcontinental e internacional. Desde una perspectiva histórica puede leerse la conexión que tienen las revistas con los acontecimientos políticos que sucedían a su alrededor y las impresiones que los sucesos formaban en toda la élite de la época. En esta clase de medios impresos se publicaron textos que tocaban temas que iban desde lo nacional hasta lo global, con ello también abrieron las perspectivas sobre estas temáticas e iniciaron el diálogo con toda América y el mundo. Esta apertura consiguió que a través de estos medios se llevaran a cabo interesantes discusiones, en donde las respuestas a un ensayo se leerían el siguiente número de la revista en cuestión, lo cual, de cierto modo, dejaba entrever la existencia de grupos, las diferencias de opinión y el carácter activo que las revistas tuvieron en el desarrollo social, político e intelectual de América Latina durante el siglo XX. Igualmente destaca la dinámica de inclusión y exclusión de autores en sus contenidos, pues muestra de manera con qué posturas y discursos había afinidades y rechazos.

La naturaleza crucial que tuvo la cultura letrada en la edición y publicación de las revistas culturales fue que generaron discusiones en torno a la unidad continental y 
sobre los atributos sociales, culturales y geográficos del subcontinente. Además, tenían la novedad que se publicaban en el continente americano a diferencia de lo que ocurrió en la segunda mitad del siglo XIX hasta la primera guerra mundial cuando las revistas culturales con temas americanos se publicaron en Europa; es el caso de publicaciones como la Revista de América, y algunas revistas contemporáneas como Cuadernos Americanos (1942), Caras y Caretas (1898), Repertorio Americano (1919), la famosa revista Sur (1931).

\section{América Latina y la cultura letrada en la Revista de América}

Ante el panorama que planteaba la segunda posguerra la Revista de América surgió como un medio por el cual era posible generar disertaciones en torno a la política y sucesos colombianos, pero también significó la construcción de un sitio para intercambiar ideas desde distintos puntos de América Latina y para reflexionar sobre el subcontinente como conjunto, así como su papel central después de las dos guerras mundiales y reflexionar sobre la relación del subcontinente con la potencia del norte de América. Es por ello que en las revistas se incluye en debate sobre lo que deparaba el futuro con respecto a los acontecimientos globales. Del mismo modo que se había planteado en la Revista de las Indias (1938), su predecesora, en la Revista de América se manifestó la preocupación por discernir cuál era el papel de la cultura y de la sociedad ante el futuro incierto, no sólo de Colombia, sino de América Latina, sobre todo ante el crecimiento exponencial que tuvo Estados Unidos tras el fin de la guerra.

De ese modo se puede leer en la sección Editorial del primer número de la revista, titulada "La Hora de América":

Se ha dicho, y es la verdad, que ésta es la hora de América. Pero lo es en dos sentidos: porque, si de un lado, aparece por la primera vez. como decisiva la actitud de nuestro hemisferio en el curso de la política mundial, por otra parte, nos acercamos a un punto en que la suerte de estas repúblicas puede jugarse como en una segunda jornada de la independencia. (Sin autor, 195: 3).

También se puede apreciar la creciente preocupación que generó entre los hombres letrados latinoamericanos la muerte de Franklin Delano Roosevelt, y la agitación que 
esta desencadenó en América Latina, pues presuponía el cambio en las políticas internacionales aplicadas desde Estados Unidos hacia América Latina, incrementando la incertidumbre generada tras los grandes conflictos bélicos.

Sobre este punto es importante recordar la importancia que tuvo la política del Buen Vecino, impulsada por Roosevelt que propició visiones diversas sobre sus repercusiones en América Latina. A grandes rasgos, esta política estaba encaminada hacia fortalecer los lazos entre las dos Américas. Por una parte, buscó favorecer el apoyo comercial de Estados Unidos sobre los territorios latinoamericanos, por el otro generar nuevas alianzas estratégicas para repeler cualquier posible ataque exterior, dejando de lado las políticas de intervención directa que habían tenido lugar décadas previas.

Por mencionar brevemente cuales fueron algunas de las medidas tomadas por Franklin D. Roosevelt con respecto a América Latina, algunas de las principales se enfocaron en la cancelación de la enmienda Platt vigente desde 1901 para favorecer una relación menos hostil con Cuba, situación que obviamente se rompió más adelante. La reacción conciliadora con respecto a la expropiación petrolera propiciada por el gobierno de Lázaro Cárdenas en México, así como se destaca la propuesta de no usar las fuerzas armadas sobre territorios en Panamá, únicamente limitarla a lo necesario en el área circundante al canal y la retirada de las tropas de marines de Haití, y los múltiples viajes que hizo durante su mandato a varios países latinoamericanos. Sin contar que uno de los ejes de la política que llevó con América latina estaba encauzada hacia los intereses de tipo económico y de coalición por medio de tratados de tipo comercial y de cooperación internacional a manera de protección del llamado "hemisferio occidental". Todas estas medidas pueden considerarse como muestras del nuevo enfoque con el cual el presidente de Estados Unidos planeaba dirigirse hacia las naciones del subcontinente. (Brewer, 2006: 103). Si bien puede decirse que en el periodo presidencial que encabezó Roosevelt se dio un auge de apoyo bilateral en el área comercial entre las dos Américas, en el campo de lo político representó el desarrollo de una política no intervencionista, incluso en ocasiones paternalista. En el rubro de lo cultural la relación se concentró en tratar de construir correspondencias y puntos de unión entre ambos territorios, destacando a la religión como el vínculo que era capaz de trascender las divergencias culturales. 
Cabe acentuar que esta proposición resultaba relativamente novedosa, en tanto que en décadas previas los mismos Gentleman Scholars se habían dado a la tarea de propagar una imagen de atraso y diferenciación racial y cultural entre la América sajona y la América hispana. (Eakin, 1998: 541-545).

Partiendo de este supuesto, la noción que Franklin D. Roosevelt trataba de plantear sobre la unidad hemisférica se tradujo también en tratar de propagar la visión del continente democrático y pacífico, en contraposición con la violencia que se daba en Europa. No obstante, a pesar de que en el discurso se manejaba la idea de democracia, en la práctica se sostuvieron procedimientos antidemocráticos. En ese sentido, gobierno estadounidense favoreció una serie de regímenes dictatoriales como el de Leónidas Trujillo que se mantuvo en el poder desde 1930-196 en República Dominicana, los Somoza en Nicaragua entre 1937 y 1979, y Fulgencio Batista en Cuba entre 1940- 1944 y 1951 a 1959, con tal de mantener el control del área del Caribe.

El ideal de una sola América representado por Roosevelt, para algunos letrados latinoamericanos no era otra cosa que un manejo de la retórica para aludir a la democracia, pero que en esencia no había cambiado la política imperialista de la potencia del norte. Estas situaciones eran interpretadas como verdaderos retrocesos en la relación bilateral entre las dos Américas. Tal proceso regresivo se incrementó tras la muerte de Roosevelt, que generó la proliferación de escenarios antipopulares, dictadura y sobre todo el incremento de posturas anticomunistas tanto en América Latina como en la región del Caribe que tuvieron lugar desde tras el arribo al poder por parte de Harry Truman, situación que más adelante agravó aún más tras el proceso revolucionario en Cuba. En textos como "Alas en las Muletas o la vida ejemplar de Roosevelt" de Octavio Méndez Pereira, puede apreciarse como se exalta la vida y trayectoria del finado presidente, así como de las políticas adoptadas hacia el subcontinente, en contraposición a la expectativa e incertidumbre que el gobierno entrante representaba. Méndez Pereira buscó hacer un homenaje a través de su escrito narrar no sólo la biografía del presidente de Estados Unidos sino a dar su punto de vista sobre sus cualidades: "Se me reveló entonces (...) como el hombre superior que había logrado alcanzar, por resortes de su vida y de su mundo íntimo, 
aquella alegría de vivir y de actuar, unida a un hondo sentido de lo justo y de lo humano". (Méndez, 1945:311).

Igualmente, Carlos Dávila en "Más allá de la política de la Buena Vecindad” expone a través de una crónica, cómo es que el nuevo presidente Truman en su visita ese mismo año a Chile, junto con el presidente chileno Juan Antonio Ríos, expusieron su interés por continuar con los fundamentos de la política de la cooperación entre las dos Américas. Dávila reseña lo ocurrido en dicha visita y cita las palabras del presidente Ríos en torno al deceso de Roosevelt y las nuevas empresas entre Estados unidos y América Latina en busca de:

(...) "solidificar la unidad americana sobre la base de los principios por los cuales se peleó y se ganó la guerra", comprendieron que la política de buena vecindad súbitamente habia avanzado un paso decisivo en su trayectoria de solidaridad continental para cimiento de la paz del mundo. (...) "Alguien dijo que poco nuevo se puede decir del Nuevo Mundo..." Yo creo que queda mucho por decir de su pasado, de su presente y, sobre todo de su futuro... Se babla de que el panamericanismo es una noción confusa de frases hechas, de que no existe aqui una tradición común ni una identidad de intereses, de aspiraciones y de modo de vida. (...) recibi yo del presidente Roosevelt testimonio de la estima cordial de este país por el mio; de cómo estas dos naciones tan distantes en lo geográfico y diferentes en los planos de la riqueza y el poder, se sentian iguales y aliadas naturales, por la identidad de sus instituciones. (...) "Durante 122 años" -agregó el presidente Ríos- "la integridad territorial de este hemisferio fue protegida por una simple declaración osada y genial; Monroe reclamó para los Estados Unidos la responsabilidad de su aplicación; Roosevelt decidió compartirla con las otras 20 naciones". (Dávila, 1945: 318-320).

Sin embargo, se conoce que los impulsos de Truman, que buscaban repeler el avance del comunismo soviético, llevaron las relaciones interamericanas por otros rumbos. De hecho, las reacciones del presidente ante el panorama posguerras, fueron muy reactivas pues se buscó bajo diversos medios contener el avance soviético, con fundamento en las propuestas de la llamada Doctrina Truman y la denominada "política al borde de guerra", que abrió la antesala del conflicto entre las dos potencias mundiales por décadas (1947-1989). (Suárez y García, 2008: 87).

Para explicar con mayor detalle cual era el carácter intercontinental, entre los países de América latina y con conexiones en otros puntos del globo, se puede comenzar explicando cómo desde la emisión del primer tomo de ésta se buscaron los medios necesarios para poder realizar ese engranaje entre autores de distintas latitudes. Es así 
como, el número 1 de la Revista de América, publicado en enero de 1945, como un esfuerzo mensual facilitado por el periódico El Tiempo', en la ciudad de Bogotá, Colombia. En su portada se logra apreciar la ilustración de Sergio Trujillo Magnenant (1911-1999), que plasmó la figura ecuestre de Simón Bolívar, seguido de siete hombres, uno de ellos le extiende una espada. El hecho de que este primer número presentara esa ilustración de Bolívar evoca la esencia misma de la integración continental que Bolívar persiguió tras el primer congreso americano de 1826, y también hace alusión a uno de los empeños que tuvo la revista, que fue conjuntar la cultura de América Latina.

Entre los textos que formaron parte del primer número pueden rescatarse varios que muestran la preocupación por tratar de desarrollar temas referentes a América Latina y su relación con otros puntos del globo. El interés de diálogo con otros personajes notables en el ámbito de las letras de otros países del subcontinente y de abordar temáticas de América en su conjunto, así como de debatir sobre los acontecimientos del momento se puede visualizar también en sus contenidos. Títulos como "Tres años de Guerra" de Carlos Dávila, "Un destino americano" de Arturo Uslar Pietri, "Carta para un ahijado de guerra" de Gabriela Mistral, "Hombres que he conocido" de Sanín Cano y "La Guerra y la Paz en 1944”, dan cuenta de la misión que engendró la Revista de América que muestra la cualidad internacional que sus fundadores trataron de impregnar en la publicación, práctica que continuó en los números sucesivos.

Igualmente, puede comenzar a revisarse cuál es la idea que se tenía sobre América Latina y su función en el acontecer global, que se ve manifiesta en el artículo "La Hora de América" en donde se aprecia que, al referirse al territorio, se alude a la unión hemisférica, a su actuar en la toma de decisiones dentro del conflicto mundial, y de las influencias que consigue tener sus decisiones en el devenir mundial. Esto se aprecia por ejemplo en la siguiente afirmación:

1 Según apunta Betancourt Mendieta, con ayuda de la directiva del periódico se le delegó la responsabilidad a Germán Arciniegas para convocar y crear una asociación de escritores que tratasen temas relativos España y América, de la cual surgió inicialmente la Revista de las Indias. Tiempo después, dadas las transformaciones que sufrió la revista, de acuerdo con las políticas nacionales, Arciniegas con ayuda del periódico El Tiempo, consiguió crear la Revista de América. véase: Betancourt, Alexander, 2019, "Un proyecto editorial colombiano en el ámbito de la Cooperación Intelectual Panamericana: la Revista de las Indias entre 1938 y 1944", (En dictamen), pp.1 a 24. 
Todo esto no es sino el principio de un despertar que hará, de boy en adelante, decisiva la actitud de las Américas para mantener la paz o conducir la guerra en el Atlántico o en el Pacífico, en Europa o en el Asia. Mañana no va a ser indiferente, ni lo es hoy, el que el gobierno de la Argentina o el de Colombia, o el de México o el de Brasil caigan en manos de un fascista o en las de un demócrata. (...) Europa se ha dado cuenta, casi exacta, y lo mismo los Estados Unidos, del poder potencial de la América Latina. (...) De esta suerte, la América Latina, en el momento de su despertar económico, queda puesta en la encrucijada de las más tremendas fuerzas internacionales que haya conocido la historia. (Sin autor, 1945: 4-5).

Las disertaciones entorno al subcontinente lo describían como un territorio natural, inhóspito, que fue heredero y transformador de la cultura occidental traída desde Europa. Sin embargo, la cultura occidental no floreció, desde este punto de vista, tras las adaptaciones culturales de la lengua, costumbres etcétera, que profundizaron el rezago, alejando a América Latina del carácter civilizatorio y moderno del legado europeo. Ante esa visión algunos de sus autores replanteaban el destino de América Latina ante los cambios que se presentaban en el orbe:

América no es en realidad parte del territorio de la cultura occidental, sino una especie de provincia transalpina de su imperio, rebelde y deformadora. (...) abandonados con los instrumentos de la civilización europea en el mundo americano, hemos vivido en un drama de adaptación que esta aun es su etapa de planteamiento. No hemos sabido encontrar el estilo de la tierra americana. América es ante todo un mundo natural, cuyo proceso cultural fue extinguido y desviado. (Uslar, 1945: 21).

Se resaltó el papel crucial de los hombres de letras en la conformación de la nueva cultura que transformase al subcontinente. De esa manera lo expresó Arturo Uslar Pietri en su texto "Un destino americano": "Ninguna empresa es más importante que la de darle expresión al espíritu de América. Los hombres de pensamiento deben esforzarse en esta angustiosa introspección para encontrar las claves sobre las cuales va a apoyarse el destino americano." (Uslar, 1945: 22).

La importancia que tuvo reflexionar sobre lo que le depararía el futuro para subcontinente tuvo un carácter crucial en los textos elaboradas por los hombres letrados. Se puede decir que fueron la manifestación de la incertidumbre que generó el periodo posguerra, la incertidumbre sobre el papel que debía tener América Latina 
con respecto a los acontecimientos globales, temor que se incrementó conforme las tensiones de la Guerra Fría iban incrementándose.

En este mismo orden de ideas, la preocupación sobre el futuro que deparaba a los territorios latinoamericanos hacía que en ocasiones circularan afirmaciones sobre el supuesto progreso al que se estaba encaminando el subcontinente así lo afirma el mismo Zulueta en su texto "América versus Europa", puesto que se observaba que tras la $2^{\circ}$ Guerra Mundial Europa estaba en aparente decadencia, mientras que se esperaba que, ante ese descenso, América Latina creciera exponencialmente. Sin embargo, dentro de las disertaciones que se pueden extraer de la Revista de América, el mismo texto de Luis de Zulueta advierte sobre los peligros u precauciones que se debían tomar con respecto a estas interpretaciones acerca del avance latinoamericano como visualizaciones muy aventuradas. Zulueta se posiciona en favor de argumentar que él percibe avances, y que probablemente el adelanto sea continuo, pero que habría que tener cuidado en afirmar que se debía exclusivamente a la debacle europea:

Sí. Yo creo que América asciende. Por muchas y muy diversas razones estoy seguro de que el próximo porvenir presenciará un rápido, esplendido engrandecimiento, material y moral, de este Nuevo Mundo. No estoy tan convencido, ni mucho menos, de la decadencia de Europa. Más, en todo caso, pienso que el problema está mal planteado cuando se emparejan el descenso de Europa y el ascenso de América como dos movimientos correlativos, necesariamente ligados entre sí, o como las dos caras, reverso y anverso, de un mismo proceso histórico. (Zulueta, 1945: 69).

Y continúa tratando de explicar con base en su interpretación del proceso de la guerra y el desastre en Europa, cómo es que América podría elevarse, aludiendo incluso a la ayuda mutua para que en términos generales existiese estabilidad global que propiciara el crecimiento americano:

No. América subirá en virtud del propio impulso, de la propia labor, de la interna fuerza ascensional. Subirá, no porque Europa baje sino sin que baje Europa, o a pesar de que Europa descienda. (...) En el fondo, necesita que Europa se Salve. Necesita un mundo equilibrado, precisamente para desarrollar en él todo su interno dinamismo, las inmensas posibilidades que hacen de América la esperanza del universo. (Zulueta, 1945: 71). 
En cuanto al tema de la cultura, las letras, el arte en América Latina, Baldomero Sanín Cano hace referencia a las influencias que Europa imprimió en América Latina. El texto "Europa en América: rumbos culturales" de cuenta de cómo es que las producciones artísticas que se generan desde América Latina tienen una fuerte relación, si no era directa sí que podía manifestarse en la educación que los hombres letrados tenían y la forma en la que ejercían la labor de escribir: "Hemos recibido de Europa el caudal de ideas y sentimientos, la lengua, las costumbres, muchos elementos de ciencia y filosofía; estamos, sumergidos unos, flotando otros, en la civilización que vino de occidente. Con todas sus fallas y chocantes deformidades esa civilización es la nuestra." (Sanín: 1945: 111).

Inclusive, Sanín Cano hace referencia directa a las influencias que Germán Arciniegas, su contemporáneo y fundador de la revista, recibió desde Europa. Sanín Cano menciona que su compatriota, a pesar de que en sus textos busca exaltar la grandeza de lo latinoamericano, no deja de ser conocimiento producido desde la apropiación que hizo el mismo Arciniegas sobre la cultura de Europa. Pero también añade que la importancia de escribir sobre lo americano y las influencias mutuas que se dan entre uno u otro continente se ven reflejadas en los intereses del autor. ${ }^{2}$ :

Nuestros lazos son estrechos, vitales e infrangibles. Uno de nuestros escritores del momento, más genuinamente colombianos y latinoamericanos es Germán Arciniegas. Su cultura, empero, es profundamente europea, quiero decir universal. Sus estudios sobre historia colonial se basan en conocimiento profundo de lo que era la vida europea en la época del descubrimiento y la Colonia y su erudición es americana de una lado y europea de los otros tres. (...) Sus libros se leen fuera de Colombia y se traducen, porque el espíritu, la actitud del autor, son europeos y universales. (Sanin, 1945: 111).

Finalmente, en el texto "América Latina en el mundo futuro" se expone cual es la preocupación inicial que favoreció la fundación y continuidad de la revista. La revista según se menciona, aun teniendo un interés genuino en fomentar la lectura, la producción de conocimiento histórico y literario también tiene un objetivo último

2 Germán Arciniegas, al igual que Sanín Cano, desarrolló un profundo empeño por hacer una apología de lo exótico y el salvajismo americano en contraposición con los estereotipos negativos que se han producido desde el viejo continente sobre América Latina. Esto se puede leer en algunos de sus textos más representativos como "América tierra firme" (1937), "Este pueblo de América" (1945) y “América en Europa” (1975), entre otros. Véase: Triviño, Consuelo, 2001, "La utopía americana de Germán Arciniegas”, en Historia Crítica, pp.1 a 6. 
que es analizar cuál es la posición de América Latina en el mundo. Es así como se menciona ese interés:

No movió a los fundadores de esta Revista tan sólo ni principalmente, el deseo de crear una nueva publicación de indole literaria, de fomentar los estudios históricos o de contribuir a propagandas culturales. (...) con una preocupación más honda por el presente y el porvenir de lo que José Martí llamara "nuestra América", quisieron que esta Revista fuera, primordialmente, un órgano de análisis y estudio de la real situación latinoamericana y de esclarecimiento de los fines esenciales que estas veintiuna repúblicas deben perseguir para establecer, claramente, cuál puede ser la misión que a ellas corresponda en la vida universal y cuáles los medios de realizarla. (Sin Autor, 1945: 321).

En ese punto, podemos añadir que efectivamente, los autores que participaban de esta publicación tenían la firme determinación de poder cambiar e intervenir en el contexto de su época, pues no se debe perder de vista la función de guías culturales y de opinión que los grupos letrados mismos asumían para incidir así entre a la gente común. (Sarlo, 1992: 12).

\section{Conclusiones}

Las revistas constituyen en sí mismas importantes fuentes para la reconstrucción de los procesos históricos de una nación. Sin embargo, su utilidad y esos van más allá de este, pues permite ver cómo es que en torno a ellas se configuró toda una serie de prácticas y mecanismos que dieron validez y legitimidad a sus contenidos, y también a las voces que hablaban a través de los textos que en ellas se publicaban. De la misma forma facilitan el análisis sobre el desarrollo de la cultura letrada y cómo fue que se utilizaron las revistas como dispositivos para llevar a cabo debates sobre los aconteceres de la época, el intercambio de ideas, la difusión literaria y también como campo de incursión política.

Ahora bien, en el interior de las revistas es posible apreciar cómo se han seleccionado algunos temas centrales a debatir que se colocan como origen y fundamento de todos los textos y discusiones que a través de ellos se generan. Las revistas entonces pueden 
caracterizarse y definirse con base en los ejes temáticos seleccionados como problemas centrales. (Sarlo, 1992: 14).

A modo de ejemplo sobre este punto, en las revistas culturales latinoamericanas que comenzaron a publicarse a partir del arielismo de Rodó, se puede apreciar todo un conjunto de valores y formas de interpretar el pasado, de rescatar y usar la memoria nacional y latinoamericana. Esto con la finalidad de interpelar a sus lectores por medio de la reflexión sobre la historia y valores que se consideraba eran capaces de unir a los territorios latinoamericanos, aludiendo al ideal una América Latina unida. En el caso específico de la Revista de América, al igual que la Revista de Indias, las áreas del conocimiento que se abordaron fueron diversas, desde temas de historia, filosofía, noticias contemporáneas, economía y poesía. También se tomó en cuenta en la revista de América a la literatura como un medio por el cual era posible afirmas la identidad del subcontinente y abogar por la unidad continental, en sus contenidos se apela entonces a la exaltación de la herencia hispánica común, el valor de lo mestizo, y la herencia cultural europea como bases de la unidad continental. Igualmente, ambas dan cuenta del quehacer escriturario y del establecimiento de un campo cultural letrado tanto colombiano como en sus conexiones fuera del país. (Betancourt, 2016, 139-140).

La Revista de América emerge como una publicación en donde sus colaboradores demuestran el creciente interés por dilucidar el devenir latinoamericano, pero también se considera como un medio de exposición de ideas, de conflagración y establecimiento de un canon escriturario que dotó a los letrados de su época con la batuta de la opinión pública. También por medio de la revista ha sido posible reflexionar que este medio se pensó como un medio de comunicación entre letrados que iba más allá de los temas de corte nacional, sino que buscó enriquecerse con los aportes tanto literarios, científicos y de opinión de toda una cúpula letrada de alto renombre a nivel internacional. Asimismo, en la revista se manifestó la preocupación por los ventos de la época y también continuó, en cierta medida la actitud repelente al imperialismo estadounidense, que se consideraba menguada con las políticas de Roosevelt. Tras su muerte, se reavivó ese sentimiento de expectativa y rechazo. Es por ello que, muchos de los esfuerzos expuestos en los textos se enfocaron en reconocer y declarar las grandezas del subcontinente, haciendo así un llamado a la 
unidad latinoamericana. A pesar de ello, la discusión con respecto al tema de la unidad continental no tomaría el suficiente auge hasta que se dio el estallido de la Revolución Cubana y el surgimiento del llamado "boom latinoamericanista" que propició a su vez retomar el valor que tenía el ejercicio de la escritura como transformadora de sentido y también como la creciente importancia de los intelectuales en la transformación social.

El presente escrito representa así un primer acercamiento a la visión sobre América Latina que trató de plasmarse en la Revista de América, sobre todo observar cómo la construcción de una idea de comunidad latinoamericana estaba sustentada en un uso específico del pasado hispánico en común. Naturalmente, este es un tema inacabado puesto que este trabajo en primer lugar hace un análisis enfocado solamente en el contexto y formulaciones sobre el primer año de vida de la revista. Para ahondar mucho más en la noción de unidad subcontinental expresada por los autores, así como la configuración de todo un sistema de cultura letrada, habrán de trabajarse otros años y otros contextos de enunciación de la publicación, así como resaltar los cambios y continuidades en aspectos relativos a la conformación y difusión de los textos, vínculos entre autores, así como de la propia idea de América Latina. Esto deja por entendido que el presente artículo deja abiertas muchas vetas más de estudio que podrán ser tratadas con mayor profundidad en futuros trabajos.

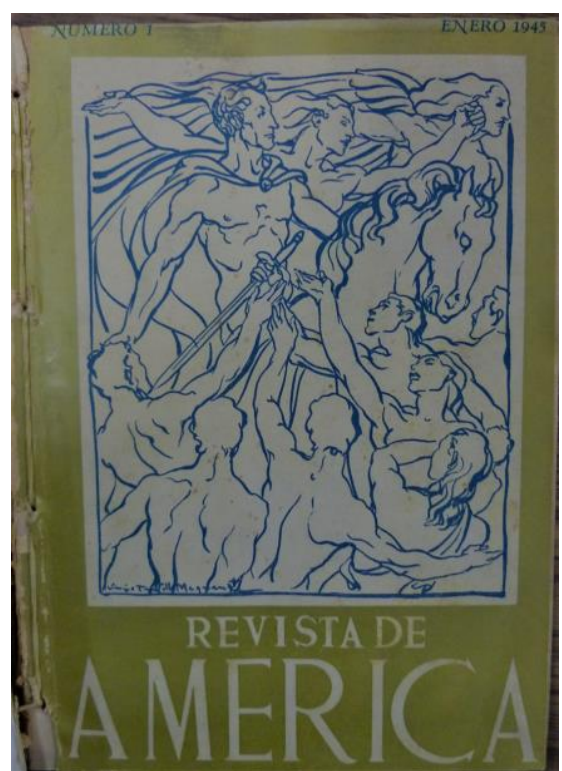

Figura 1. Portada Revista de América, Núm. 1, Vol. 1, 1945. Ilustración de Simón Bolívar a cargo de Sergio Trujillo Magnenant. 


\section{FUENTES}

Dávila, C. 1945, "Más allá de la política de Buena Vecindad" en Revista de América, Núm. 11, Vol. 4, pp. 318 a 320.

Mendez, O. 1945, "Alas en las muletas o la vida ejemplar de Roosevelt" en Revista de América, Núm. 11, Vol. 4, pp. 311 a 312.

Sanín, B. 1945, "Europa en América: rumbos culturales" en Revista de América, Núm. 7, Vol. 3, pp. 108 a 112.

Sin autor. 1945, “América Latina en el mundo futuro" en Revista de América, Núm. 3, Vol. 1, pp. 321 a 323.

Sin autor. 1945, "Editorial: La Hora de América" en Revista de Ámerica, Núm. 1, Vol. 1 , pp. 3 a 10.

Uslar, A. 1945, "Un destino americano" en Revista de Ámerica, Núm. 1, Vol. 1, pp. 20 a 22.

Zulueta, L. 1945, “América versus Europa” en Revista de América, Núm. 4, Vol. 2, pp. 69 a 71.

\section{BIBLIOGRAFÍA}

Altamirano, C. 2008, "Introducción" en Historia de los intelectuales en América Latina, Buenos Aires, Katz, pp. 9 a 28.

Beigel, F. 2003, "Las revistas culturales como documentos de la historia latinoamericana" en Utopia y Praxis Latinoamericana, Vol. 8, Núm. 20, pp. 105 a 115.

Betancourt, A. 2013, "La perspectiva continental: entre la unidad nacional y la unidad de América Latina" en Historia Crítica, Núm. 49, pp. 135 a 157.

Betancourt, A. 2016, "Revista de Indias: (19381950): la difusión cultural y el mundo letrado" en Anuario de historia regional y de las fronteras, Vol. 21-2.

Betancourt, A. 2018, América Latina: cultura letrada y escritura de la historia, Anthropos/Siglo XXI, Barcelona/ México.

Betancourt, A. 2019, "Un proyecto editorial colombiano en el ámbito de la Cooperación Intelectual Panamericana: la Revista de las Indias entre 1938 y 1944", (En dictamen), pp.1 a 24.

Betancourt, A. 2021, "La profesionalización de la historia en Colombia. Jaime Jaramillo Uribe: contextos, trayectoria y corrientes historiográficas" en Anuario Colombiano de Historia Social y de la Cultura, Vol. 48, Núm. 1, pp. 231 a 255 .
Botinelli, A. \& Sanhueza, M. 2019, "Literatura y política en América Latina en el siglo XX: apuntes para una discusión. Introducción" en Pléyade, Núm. 24, pp. 21 a 47.

Brewer, S. 2006, Borders and Bridges: A bistory of U.S.-Latin American relations, Praeger Security International, Westport- Connecticut.

Deves, E. 1997, “El pensamiento latinoamericano a comienzos del siglo XX: la reivindicación de la identidad" en Cuyo, Anuario de Filosofía Argentina y Americana, Núm. 14, pp. 11 a 75.

Eakin, M. 1998, "Latin American History in the United States: From Gentlemen Scholars to Academic Specialists" en The History Teacher, Vol. 31, Núm. 4, pp. 539 a 561.

Granados, A. 2017, "Las Redes Intelectuales Latinoamericanas en perspectiva historiográfica: una mirada desde México" en Historia y Espacio, Vol. 13, Núm. 49, pp. 63 a 95.

Guzmán, D. \& Marín, P. 2016, "Lectores y textos escolares durante la primera mitad del siglo XX en Colombia" en La palabra, Núm. 29, pp. 185 a 197.

Mallosetti, L. 2010, “Arte e historia en los festejos del Centenario de la Revolución de mayo en Buenos Aires" en Historia Mexicana, Vol. LX, Núm. 1, pp. 439 a 471.

Pita, A. \& Grillo, M. 2015, "Una propuesta de análisis para el estudio de revistas culturales", en Revista Latinoamericana de Metodología de las Ciencias Sociales, Vol. 5, Núm. 1, pp. 1-30.

Sábato, H. 2008, "Nuevos espacios de formación y actuación intelectual: prensa, asociaciones, esfera pública (1850-1900)" en Altamirano, C. Historia de los intelectuales en América Latina, Katz, Buenos Aires, pp. 387 a 411.

Sarlo, B. 1992, "Intelectuales y revista: razones de una práctica" en América: Cahiers du CRICCAL, Núm. 9-10, Le discours culturel dans les revues latino-américaines, 1940-1970, pp. 9 a 16.

Suárez, L. \& García, T. 2008, "Lección 5: Las relaciones interamericanas durante la "Época del Buen vecino" en Las relaciones interamericanas: continuidades y cambios, Consejo Latinoamericano de Ciencias Sociales CLACSO, Buenos Aires.

Triviño, C. 2001, "La utopía americana de Germán Arciniegas" en Historia Crítica, pp. 1 a 6. 